\title{
Signal Reconstruction with Semidefinite Relaxation Optimization in Uplink Massive MIMO Systems
}

Guo Li ( $\sim$ gli@xidian.edu.cn )

Xidian University

\section{Research}

Keywords: Massive MIMO system, signal reconstruction, semidefinite relaxation optimization, uplink transmission, BER performance

Posted Date: July 27th, 2020

DOl: https://doi.org/10.21203/rs.3.rs-44859/v1

License: (9) This work is licensed under a Creative Commons Attribution 4.0 International License. Read Full License

Version of Record: A version of this preprint was published at IEEE Communications Letters on January 1st, 2021. See the published version at https://doi.org/10.1109/LCOMM.2020.3025633. 


\title{
Signal Reconstruction with Semidefinite Relaxation Optimization in Uplink Massive MIMO Systems
}

\author{
Guo Li \\ Correspondence: gli@xidian.edu.cn \\ Xidian University, 2 South Taibai Road, 710071, Xi'an, P. R. China
}

\begin{abstract}
In this paper, we consider a single group massive MIMO system, which consists of one receiver (base station) deployed with a large number of antennas and multiple users with each having single antenna. In the uplink transmissions from users to base station, we focus on an efficient signal reconstruction of all the users' transmitted symbols. We build a statistic decision matrix based on the received signals, whose eigenvectors and eigenvalues constitute the key components of our signal reconstruction algorithm. Then, an optimization problem is formulated and we convert this problem to two subproblems: obtaining the optimal weights by solving Semidefinite Relaxation (SDR) optimization and obtaining the optimal rotation phases. Finally, an iterative algorithm framework is proposed for multi-user signal reconstruction. Numerical results are also carried out to verify the bit-error-ratio (BER) performance compared with the several coherent detection schemes and the noncoherent transmission schemes.
\end{abstract}

\section{Keyword}

Massive MIMO system, signal reconstruction, semidefinite relaxation optimization, uplink transmission, BER performance

\section{Introduction}

\subsection{Massive MIMO and Motivation}

Massive multiple-input multiple-output (MIMO) [1,2] is a hot topic research in recent years and has been considered as one promising technology for the future wireless communication systems, since it can provide many benefits, such as great 
improvements in throughput, efficient radiated energy and simplification of the MAC layer [3]. Essentially, these performance improvements mainly result from the higher beamforming gain at transmitter or diversity gain at receiver due to the large number of independent channel realizations of the active antennas.

Generally, in order to ensure a high receiver performance, the demodulator must know the channel state information (CSI), which is estimated before demodulation and decoding procedures $[4,5]$. Therefore, training sequences or pilot signals are usually embedded in the transmission frame structures to obtain the channel knowledge. However, the acquisition of the whole accurate instantaneous CSI is not an easy thing for the massive MIMO systems. The cost of time and frequency resources significantly increase with the number of the active antennas and users. In cellular scenarios [1], a frequency-division duplex (FDD) system generally requires large amount of pilots which scales linearly with the number of antennas. Even in time-division duplex (TDD) mode, since the circuits in transmitting and receiving paths could result in different drifting, a large number of training sequences need to make calibration in order to maintain the channel reciprocity [6]. In long term evolution (LTE) [7], the channel estimation and pilot overhead occupy approximately $\$ 151 \% \$$ amount of time and frequency resources. These investigations suggest that the channel training in coherent system may be inefficient, especially in massive-antenna scenarios.

CSI acquisition is generally necessary when the receiver adopts the coherent detection, such as the commonly-used maximal ratio combining (MRC) algorithm. In the uplink transmissions, the CSI estimations at the base station are obtained from the signals transmitted by the multiple users in TDD mode. In FDD mode, the CSI estimations are obtained with the CSI feedback from the multiple users by using the different transmission frequency. The CSI plays an important role in the receiver algorithm, such as the signal equalization. In addition, the transmission schemes without the CSI training or estimation become more necessary and practical, especially in the internet of things (IoT), device-to-device (D2D) or massive MIMO scenarios. The most noncoherent transmission schemes focus on elaborately designing the transmitting signals. 
Motivation: In this paper, we expect to reduce the cost of pilots or training sequences as much as possible in an approximately non-coherent transmission. We alternatively turn our attention on an efficient signal processing and signal reconstruction at the receiver.

\subsection{Related Works}

The perception and acquisition of the CSI are crucial for single-input-single-output (SISO) and multiple-input-multiple output (MIMO) systems. Generally, the channel training is commonly used in the current practical systems. However, in the massive MIMO systems, the large number of antenna elements induce two major challenges. The one is a large number of radio frequency (RF) chains increases the energy consumptions. The other is RF chains occupy an increasing amount of spectral resources. Therefore, several improved transmission schemes and CSI acquisition strategies are proposed in order to reduce the processing complexity. Hybrid beamforming [8] uses a combination of analog beamformers in the RF domain, together with digital beamforming in the baseband, so as to reduce the RF with a smaller number of up/down-conversion chains. Joint spatial division and multiplexing (JSDM) proposed in $[9,10]$ provides significant potential to achieve link gains in massive MIMO systems. Its key idea lies in partitioning the users into groups with approximately similar covariance matrices and splitting beamforming into a pre-beamforming and the multi-user MIMO (MU-MIMO) precoder, which results in the significantly reduced training dimensions. In addition, the beam division multiple access transmission proposed in [11] with the similar idea to simultaneously serve multiple users via different beams. The sparsity property and the phase-only precoding $[12,13]$ significantly reduce the overhead of channel estimation.

In order to avoid the channel estimation before signal detection at the receiver, the noncoherent signal processing provides the ideal choice. Actually, noncoherent unitary space-time transmissions in multi-antenna system have been intensively considered [14-16]. In massive MIMO systems, it is crucial to fully utilize the receiving freedom of the receiver's large-scale antennas in order to design the noncoherent transmission 
strategy. The unitary space-time modulation $[17,18]$ was proposed earlier for avoiding the need of CSI estimation at the receiver. However, this scheme was designed for the classical MIMO systems and showed to be effective for the long coherence time intervals and for the high signal-to-noise ratio (SNR) scenarios. An energy-detectionbased noncoherent communication scheme for multiuser massive single-input multipleoutput (SIMO) systems with multiple access channels (MAC) was proposed in [19-21], where an Amplitude Shift Keying (ASK) modulation was described. This scheme showed that in terms of a scaling law of achievable rate, the performance of the energybased noncoherent transmission is not different from that with perfect CSI at transmitters and receivers. However, the transmitted symbols in this scheme are limited to the amplitude signals, which generally requires excessive antennas at receiver for the considerable performance. A differential Phase Shift Keying (PSK) aided large-scale MIMO system was designed in [22], which proposed a transmitting constellation design by allocating different power factors to different transmitters. These power factors are not straightforward for general PSK constellations, which generally need exhaustive search to determine their optimal values. To design well noncoherent transmitting signal structure, authors in [23] proposed a $2 \times 2$ noncoherent space-time signal block in point-to-point massive MIMO system, which does not require any knowledge of instantaneous CSI at either transmitter or receiver. Furthermore, the determinant and trace criteria of this noncoherent signal structure were also presented in [23] based on the noncoherent maximum likelihood detector. In addition, a transmission scheme based on uniquely factorable constellation (UFC) is proposed [24], which enables transmission without knowing the channel information.

\subsection{Contributions}

The main contributions of this paper can be summarized as

- Firstly, a statistic decision matrix is built based on the received signals, whose eigenvectors and eigenvalues constitute the key components of our signal reconstruction algorithm.

- Then, an optimization problem is refined to reconstruct one user's transmitted 
signals. Then, we convert this problem to two equivalent sub-problems, i.e., obtain the optimal weights by solving Semidefinite Relaxation (SDR) optimization and obtain the optimal rotation phases.

- Finally, an iterative algorithm framework is proposed for multi-user signal reconstruction. The overall complexity analyses are also included.

\subsection{Paper structure}

The rests of this paper are organized as follows. We present the system and transmission model in Section 2.1. The basic signal reconstruction model is described in section 2.2. In section 3, a reconstruction algorithm with SDR optimization is formulated and the complete iterative algorithm is presented. Simulation results and conclusions are then shown in section 4 and section 5 respectively.

Notations: Bold lowercase $\mathbf{x}$ and bold uppercase $\mathbf{A}$ denote vector and matrix respectively. We use $\mathbf{A}^{T}, \mathbf{A}^{\dagger}, \mathbf{A}^{H}$, $\operatorname{tr} \mathbf{A}$ and rank $\mathbf{A}$ to denote the transpose, conjugate, Hermitian transpose, trace and rank of matrix A respectively; $\mathcal{C N}(\mathbf{0}, \mathbf{X})$ denotes the complex Gaussian distribution with zeros mean and covariance matrix $\mathbf{X}$. $\mathbb{R}^{+}$denotes the set of positive real numbers and $\mathbb{H}^{K}$ denotes the set of $K \times K$ Hermitian matrices. $\mathbf{I}_{K}$ and $\mathbf{0}_{K}$ denotes the $K \times K$ identity matrix and all zeroelement matrix.

\section{Problem formulation}

\subsection{System and Transmission Model}

As shown in Figure 1, we consider $K$ single-antenna transmitters (Users) and one receiver (Base-station) deployed with $N$ antennas, $N \gg K$. This paper focuses on the uplink transmission where all the users transmit their symbols simultaneously and independently. We assume no accurate channel state information (CSI) is available at either the users or the base station. A quasi-static channel fading with channel coherence time $\mathcal{T}$ can be modeled as $\mathbf{h}_{k}=\left[h_{k, 1}, h_{k, 2}, \cdots, h_{k, N}\right]^{T}$, where $h_{k, n}$ denotes the fading 
coefficient between the $k$-th users to the $n$-th antenna of the base station, $k=1,2, \cdots, K, n=1,2, \cdots, N$. Generally, the channel fading undergoes large-scale and small-scale fading, that is to say $h_{k, n}=\sqrt{\beta_{k}} \bar{h}_{k, n}$, where $\beta_{k}$ denotes the large-scale fading and $\bar{h}_{k, n}$ denotes the small-scale fading. In typical scenarios, the user's physical position changes negligibly in one observation duration. Hence, the large-scale fading parameter $\beta_{k}$ can be treated as unchanged within one channel coherence time $\mathcal{T}$. We model as the random variable with independent and identical distributed (i.i.d.) complex Gaussian $\mathcal{C N}(0,1)$. It points out that the spatial characteristics vary in the order of seconds, while the sampling duration is usually in the order of microsecond.

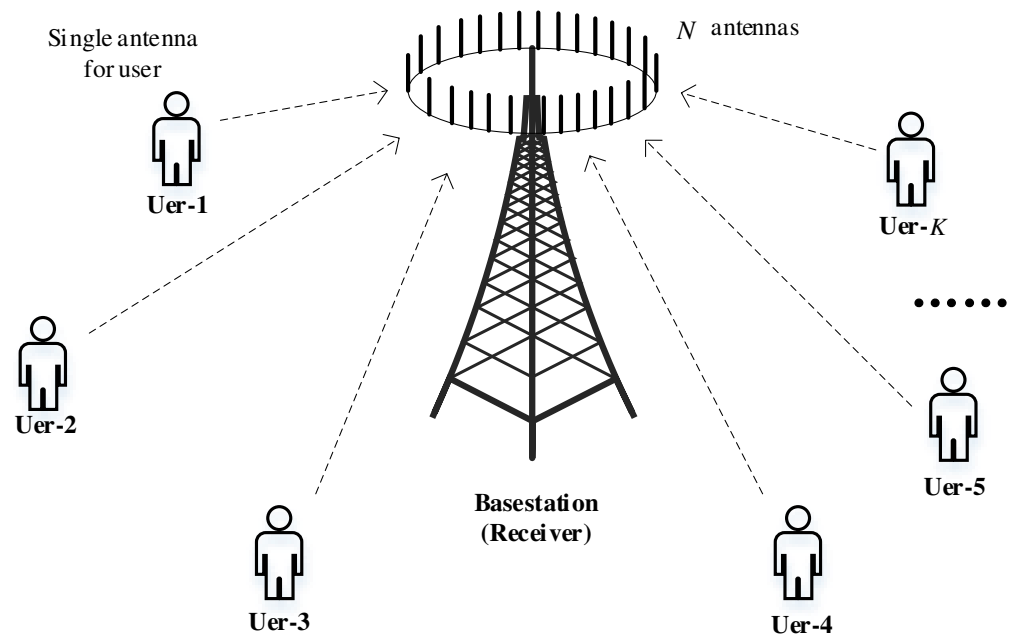

Figure 1: Scenario with multiple users and a based station having large number of antennas

The received signals at every receiving antenna are sampled according to the system $\mathrm{AD} / \mathrm{DA}$ modules after the matched filters. Let the $1 \times L$ row vector $\mathbf{s}_{k}=\left[s_{k, 1}, s_{k, 2}, \cdots, s_{k, L}\right]$, be the transmitted symbols from the $k$-th user during the $L$ time slots, $L<\mathcal{T}$, in which $s_{k, l}$ is selected from a power-normalized constellation, $k=1,2, \cdots, K, l=1,2, \cdots, L$. Then, during the $l$-th time slot, the received signal at the base station can be expressed as

$$
\mathbf{y}_{l}=\sum_{k=1}^{K} \sqrt{P_{k}} \mathbf{h}_{k} s_{k, l}+\boldsymbol{\xi}_{l},
$$

where $P_{k}$ denotes the transmitting power of the $k$-th user, $\xi_{l}$ denotes the noise 
vector with each element having i.i.d. complex Gaussian distribution with zero mean and noise power $\sigma^{2}$.

After the $L$ observations at base station, the whole received signals can be compactly combined as

$$
\mathbf{Y}=\left[\mathbf{y}_{1}, \mathbf{y}_{2}, \cdots, \mathbf{y}_{L}\right]=\mathbf{H} \mathbf{\Lambda}_{P} \mathbf{S}+\boldsymbol{\Xi},
$$

where $\mathbf{H}=\left[\mathbf{h}_{1}, \mathbf{h}_{2}, \cdots, \mathbf{h}_{K}\right]$ with dimension $N \times K$ denotes the equivalent channel matrix, $\quad \boldsymbol{\Lambda}_{P}=\operatorname{diag}\left(\sqrt{P_{1}}, \sqrt{P_{2}}, \cdots \sqrt{P_{K}}\right) \otimes \mathbf{I}_{N}$ denotes the diagonal power matrix, $\mathbf{S}=\left[\mathbf{s}_{1}, \mathbf{S}_{2}, \cdots, \mathbf{S}_{K}\right]^{T}$ with dimension $K \times L$ denotes the combined transmitted symbol matrix of all the users, $\boldsymbol{\Xi}=\left[\boldsymbol{\xi}_{1}, \boldsymbol{\xi}_{2}, \cdots, \boldsymbol{\xi}_{L}\right]$ denotes the additive complex Gaussian noises.

Considering the large-scale fading $\beta_{k}$ and small fading $\overline{\mathbf{h}}_{k}$, we express the equivalent channel vector as $\mathbf{H}=\overline{\mathbf{H}} \boldsymbol{\beta}$, where $\boldsymbol{\beta}=\operatorname{diag}\left(\sqrt{\beta_{1}}, \sqrt{\beta_{2}}, \cdots \sqrt{\beta_{K}}\right)$, $\overline{\mathbf{H}}=\left[\overline{\mathbf{h}}_{1}, \overline{\mathbf{h}}_{2}, \cdots, \overline{\mathbf{h}}_{K}\right]$ and $\overline{\mathbf{h}}_{k}=\left[\bar{h}_{k, 1}, \bar{h}_{k, 2}, \cdots, \bar{h}_{k, N}\right]^{T}$

In order to overcome the influence of large-scale fading on received signal average energy, transmitting power is allocated as $P_{k}=c^{2} / \beta_{k}$, where $c$ is a constant. Then, we have $\boldsymbol{\Lambda}_{P} \boldsymbol{\beta}=c \mathbf{I}_{K}$. The transceiver model in (2) can be rewritten as $\mathbf{Y}=c \overline{\mathbf{H}} \mathbf{S}+\mathbf{\Xi}$.

It is worth noting that the received signals in (2) has the dimension $N \times L$, where $N$ is largely greater than $K$ in our massive MIMO scenario. That is to say, benefiting from the large degree of freedom (DoF) of antenna array at the base station, it is possible for us to build a detector to extract the transmitted symbols directly from the received signals without extra operations for channel information acquisition.

\section{2 signal Reconstruction with Statistical Eigenvectors}

Preliminarily, we first introduce several useful properties of very large antenna arrays. Assuming $\mathbf{p}$ and $\mathbf{q}$ to be two $N \times 1$ mutually independent vectors whose elements are i.i.d. zero-mean complex Gaussian random variables with variances of $\sigma_{p}^{2}$ and $\sigma_{q}^{2}$ 
respectively. According to the probability statistical theory [25], we have

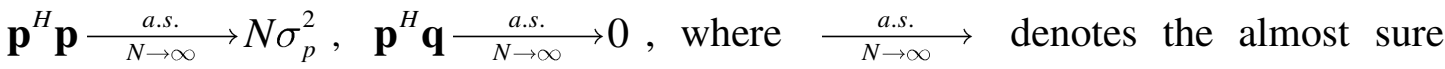
convergence in distribution when $\$ \mathrm{~N} \$$ approaches to the infinity.

Therefore, in our transmission model (2), we have $\overline{\mathbf{H}}^{H} \overline{\mathbf{H}} / N \underset{N \rightarrow \infty}{\stackrel{\text { a.s. }}{\longrightarrow}} \mathbf{I}_{K}$,

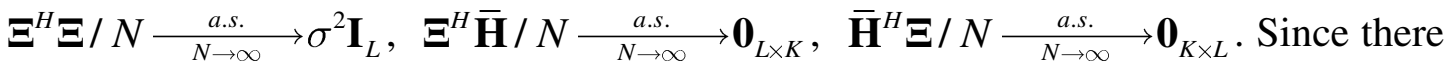
is no accurate CSI known at base station, we just build a new \$LItimes L $\$$ statistic decision matrix as

$$
\begin{aligned}
& \boldsymbol{\Sigma}=\frac{1}{N} \mathbf{Y}^{H} \mathbf{Y}, \\
= & c^{2} \mathbf{S}^{H} \frac{\overline{\mathbf{H}}^{H} \overline{\mathbf{H}}}{N} \mathbf{S}+\frac{c}{N} \mathbf{S}^{H} \overline{\mathbf{H}}^{H} \boldsymbol{\Xi}+\boldsymbol{\Xi}^{H} \overline{\mathbf{H}} \mathbf{S}+\frac{\boldsymbol{\Xi}^{H} \boldsymbol{\Xi}}{N},
\end{aligned}
$$

Combined with (2), the asymptotic expression of this statistic decision matrix, when $N$ is very large, can be expressed as

$$
\boldsymbol{\Sigma}_{N \rightarrow \infty}=c^{2} \mathbf{S}^{H} \mathbf{S}+\sigma^{2} \mathbf{I}_{L}
$$

Clearly we observe that $\boldsymbol{\Sigma}_{N \rightarrow \infty}$ contains no channel fading gains but the only transmitted symbol matrix $\mathbf{S}$ and the statistical noise power $\sigma^{2}$. Therefore, it means we can obtain the symbol estimations regardless of the channel coefficients.

In this paper, we expect to propose a signal reconstruction method based on the decision statistic matrix $\Sigma$. Before to do that, we consider the noise-free case as the exploratory investigation. Based on (5), we can easily obtain the noise-free statistic decision matrix as $\boldsymbol{\Sigma}_{N \rightarrow \infty}^{0}=c^{2}\left(\mathbf{s}_{1}^{H} \mathbf{s}_{1}+\mathbf{s}_{2}^{H} \mathbf{s}_{2} \cdots+\mathbf{s}_{K}^{H} \mathbf{s}_{K}\right)$. Due to $\mathbf{S}=\left[\mathbf{s}_{1}, \mathbf{s}_{2}, \cdots, \mathbf{s}_{K}\right]^{T}$, the structure of $\boldsymbol{\Sigma}_{N \rightarrow \infty}^{0}$ suggests that it is possible to extract the individual symbols $\mathbf{s}_{k}, k=1,2, \cdots, K$. Since $\boldsymbol{\Sigma}_{N \rightarrow \infty}^{0}$ is a Hermitian symmetric matrix, it can be decomposed as $\boldsymbol{\Sigma}_{N \rightarrow \infty}^{0}=\mathbf{U} \boldsymbol{\Lambda} \mathbf{U}^{H}$, where $\mathbf{U}$ denotes the $L \times L$ unitary matrix consisting of the eigenvectors, and the $\boldsymbol{\Lambda}$ is the corresponding diagonal matrix consisting of the eigenvalues. Furthermore, because the rank of $\boldsymbol{\Sigma}_{N \rightarrow \infty}^{0}$ is $K$, it has only $K$ nonzero eigenvalues. Therefore, the transmitted symbols $\mathbf{S}$ can be 
reconstructed from these eigenvalues and eigenvectors

$$
\mathbf{S}=\mathbf{\Theta Q} \mathbf{\Lambda}^{\frac{1}{2}} \mathbf{U}^{H}
$$

where $\tilde{\boldsymbol{\Lambda}}=\operatorname{diag} \lambda_{1}, \lambda_{2}, \cdots, \lambda_{K} \quad$ with $\lambda_{i}$ being the eigenvalue, $\tilde{\mathbf{U}}=\left[\mathbf{u}_{1}, \mathbf{u}_{2}, \cdots, \mathbf{u}_{K}\right]$ with $\mathbf{u}_{i}$ being the eigenvector corresponding to the eigenvalue $\lambda_{i}, i=1,2, \cdots, K, \mathbf{Q}$ is an unitary matrix, i.e., $\quad \mathbf{Q Q}^{H}=\mathbf{I}_{K}, \quad \boldsymbol{\Theta}=\operatorname{diag} e^{j \theta_{1}}, e^{j \theta_{2}}, \cdots, e^{j \theta_{K}}$, $\theta_{k} \in 0,2 \pi, k=1,2, \cdots, K$. Here, $\mathbf{Q}$ reflects the linear relationship between the $K$ eigenvectors and the element $e^{j \theta_{k}}$ in $\boldsymbol{\Theta}$ reflects the phase rotation. This reconstruction method can be verified by performing $\left(\boldsymbol{\Theta} \mathbf{Q} \tilde{\mathbf{\Lambda}}^{\frac{1}{2}} \tilde{\mathbf{U}}^{H}\right)^{H} \mathbf{\Theta Q} \tilde{\mathbf{\Lambda}}^{\frac{1}{2}} \tilde{\mathbf{U}}^{H}=\boldsymbol{\Sigma}_{N \rightarrow \infty}^{0}$.

Given the $L \times L$ statistic decision matrix in (3) and (4), we can refer to the noisefree and infinite $N$ case, i.e., $\boldsymbol{\Sigma}_{N \rightarrow \infty}^{0}$. Since the noise terms in (4) cannot be ignored, the results and analyses will not be valid in (6). Due to the nonlinear nature of Eigen decomposition, it is difficult to give precise analysis of the effect of noise terms on the Eigen properties of the matrix. However, we can also perform the reconstruction processes with similar idea. We observe that the term $\mathbf{H}^{H} \mathbf{H} / N$ in (4) is a diagonaldominant matrix, and the term $\left(\mathbf{S}^{H} \mathbf{H}^{H} \boldsymbol{\Xi}+\boldsymbol{\Xi}^{H} \mathbf{H} \mathbf{S}+\boldsymbol{\Xi}^{H} \boldsymbol{\Xi}\right) / N$ goes to very small values resulting from the independence between random variable $\mathbf{H}$ and $\mathbf{\Xi}$. That is to say there must be $K$ nonzero eigenvalues clearly larger than the others among all the $L$ eigenvalues of $\boldsymbol{\Sigma}$ in (3). Therefore, we just focus on the dominant components of these eigenvalues.

Based on the above analyses, the $k$-th user's estimation can be reconstructed as

$$
\hat{\mathbf{s}}_{k}=e^{j \theta_{k}} \sum_{i=1}^{K} \alpha_{k, i} \sqrt{\lambda_{i}} \mathbf{u}_{i}^{H}
$$

where $\lambda_{i}$ is the eigenvalue of the $L \times L$ decision statistic matrix $\boldsymbol{\Sigma}$ in (3), $\mathbf{u}_{i}$ denotes the eigenvector with $\Sigma \mathbf{u}_{i}=\lambda_{i} \mathbf{u}_{i}, \theta_{k} \in 0,2 \pi$. Certainly, the parameters $\alpha_{k, i}$ and $\theta_{k}$ are needed to be determined according to some metric. From [7], we know the $k$-th user's estimation $\hat{\mathbf{s}}_{k}$ is related with parameters $\alpha_{k, i}$ and $\theta_{k}$. Here, we could define a metric function $\varepsilon \alpha_{k, 1}, \alpha_{k, 2}, \cdots, \alpha_{k, K}, \theta_{k}$ with the average symbol energy 
based on the assumption of the normalized average power of the transmitted symbols,

$$
\varepsilon \alpha_{k, 1}, \alpha_{k, 2}, \cdots, \alpha_{k, K}, \theta_{k}=\left.\sum_{l=1}^{L}|| \hat{\mathbf{s}}_{k}[l]\right|^{2}-\left.\chi\right|^{2} \text {, }
$$

where $\hat{\mathbf{s}}_{k}[l]$ denotes the $l$-th element of vector $\hat{\mathbf{s}}_{k}, \chi$ means the expected average power. The optimal values of parameters $\alpha_{k, i}$ and $\theta_{k}$ should make the reconstructed symbols $\hat{\mathbf{s}}_{k}$ minimizing the metric function $\varepsilon \alpha_{k, 1}, \alpha_{k, 2}, \cdots, \alpha_{k, K}, \theta_{k}$. From (6), we have already know the $k, i$-th element $\alpha_{k, i}$ of the unitary matrix $\mathbf{Q}$ should satisfy $\left|\alpha_{k, 1}\right|^{2}+\left|\alpha_{k, 2}\right|^{2}+\cdots+\left|\alpha_{k, K}\right|^{2}=1$. Hence, the optimal parameters can be obtained with solving the following optimization problem

$$
\begin{gathered}
\text { (P1) } \min \varepsilon \alpha_{k, 1}, \alpha_{k, 2}, \cdots, \alpha_{k, K}, \theta_{k} \\
\text { s.t. } \quad \sum_{i=1}^{K} \alpha_{k, i} \alpha_{k^{\prime}, i}^{\dagger}= \begin{cases}1, & k=k^{\prime} \\
0, & k \neq k\end{cases} \\
\theta_{k} \in 0,2 \pi
\end{gathered}
$$

We observe it's very computationally complex to obtain the whole $K K+1$ optimal parameters according the above optimization problem (P1), especially when $K$ is large. More efficient algorithm will be designed in the next section.

\section{Method: reconstruction Algorithm with SDR Optimization}

Carefully, we observe that the value of $\theta_{k}$ in (7) and (8) cannot affect the value of $\left|\hat{\mathbf{s}}_{k}[l]\right|^{2}$. Therefore, we temporarily take away the parameter $\theta_{k}$. Let

$$
\tilde{\mathbf{s}}_{k}^{T}=\sum_{i=1}^{K} \alpha_{k, i} \sqrt{\lambda_{i}} \mathbf{u}_{i}^{H}=\mathbf{x}_{k}^{T} \tilde{\mathbf{\Lambda}}^{\frac{1}{2}} \tilde{\mathbf{U}}^{H}
$$

where $\quad \mathbf{x}_{k}=\left[\alpha_{k, 1}, \alpha_{k, 2}, \cdots, \alpha_{k, K}\right]^{T}$, the diagonal matrix $\tilde{\boldsymbol{\Lambda}}^{\frac{1}{2}}=\operatorname{diag} \sqrt{\lambda_{1}}, \sqrt{\lambda_{2}}, \cdots$, $\sqrt{\lambda_{i}}, \cdots, \sqrt{\lambda_{K}}$ with $\lambda_{i}$ being the first $K$ eigenvalues of the $L \times L$ decision statistic matrix $\Sigma, \lambda_{1} \geq \lambda_{2} \geq \cdots \geq \lambda_{K}, \quad \mathbf{u}_{i}$ is the eigenvector corresponding to $\lambda_{i}$. In order to give a convenient representation, we define $\quad \mathbf{V}=\tilde{\boldsymbol{\Lambda}}^{\frac{1}{2}} \tilde{\mathbf{U}}^{H}$ and rewrite $\mathbf{V}$ 
with $L$ column vectors, i.e.,

$$
\mathbf{V}=\tilde{\mathbf{\Lambda}}^{\frac{1}{2}} \tilde{\mathbf{U}}^{H}=\left[\mathbf{v}_{1}, \mathbf{v}_{2}, \cdots, \mathbf{v}_{L}\right]
$$

\subsection{Reconstruction of the transmitted symbols using SDR optimization}

As for the optimization (P1), it is not easy to solve this problem directly. Alternatively, we find that a problem of minimizing a summation can be converted to a problem of minimizing the maximum of the summands. Hence, the problem (P1) can be reformulated as

$$
\begin{gathered}
\left.(\mathrm{P} 2) \quad \min _{l=1,2, \cdots, L} \max _{1}|| \tilde{\mathbf{s}}_{i}[l]\right|^{2}-\chi \mid \\
\text { s.t. } \quad \mathbf{x}_{k}^{H} \mathbf{x}_{k}=1
\end{gathered}
$$

Although (P2) takes away the summation of (P1), it still belongs to the class of NPhard problems [26]. However, we can see $\tilde{\mathbf{s}}_{i}[l]=\mathbf{x}_{k}^{T} \mathbf{v}_{l}$ and

$$
\left|\tilde{\mathbf{s}}_{i}[l]\right|^{2}=\mathbf{x}_{k}^{T} \mathbf{v}_{l} \mathbf{v}_{l}^{H} \mathbf{x}_{k}^{\dagger}=\operatorname{tr} \mathbf{v}_{l} \mathbf{v}_{l}^{H} \mathbf{x}_{k}^{\dagger} \mathbf{x}_{k}^{T}
$$

Here, we notice $\mathbf{x}_{k}^{\dagger} \mathbf{x}_{k}^{T}$ is a $L \times L$ matrix and $\operatorname{rank}\left(\mathbf{x}_{k}^{\dagger} \mathbf{x}_{k}^{T}\right)=1$. Clearly, this is a nonconvex Quadratically Constrained Quadratic Programs (QCQPS). According to [26], this problem can be efficiently solved by Semidefinite Relaxation (SDR). Therefore, let $\mathbf{X}_{k}=\mathbf{x}_{k}^{\dagger} \mathbf{x}_{k}^{T}$, the problem (P2) can be reformulated as

$$
\begin{aligned}
& \text { (P3) } \min _{t \in \mathbb{R}^{+}, \mathbf{X} \in \mathbb{H}^{K}} t \\
& \text { s.t. } \quad-t \leq \operatorname{tr} \mathbf{v}_{l} \mathbf{v}_{l}^{H} \mathbf{X}_{k}-\chi \leq t \\
& \operatorname{tr} \mathbf{X}_{k}=1, \mathbf{X}_{k} \succeq 0 \\
& \quad l=1,2, \cdots, L
\end{aligned}
$$

This optimization problem can be efficiently solved by using the cvx toolbox to obtain the global optimal solution $\mathbf{X}_{k}^{*}$.

The final procedure of using SDR is how to convert a globally optimal solution $\mathbf{X}_{k}^{*}$ 
into our expected solution. Generally speaking, the rank of this globally optimal solution $\mathbf{X}_{k}^{*}$ is larger than one, that is to say we must somehow extract a vector feasible for (12). There are two methods to do this extraction. The one is to apply a rank-one approximation on $\mathbf{X}_{k}^{*}$. Specially, let $r=\operatorname{rank}\left(\mathbf{X}_{k}^{*}\right)$, and then $\mathbf{X}_{k}^{*}=\sum_{i=1}^{r} \lambda_{i}^{\prime} \mathbf{q}_{i} \mathbf{q}_{i}^{H}$, where $\lambda_{1}^{\prime} \geq \lambda_{2}^{\prime} \geq \cdots \geq \lambda_{r}^{\prime}>0$ and $\mathbf{q}_{1}, \mathbf{q}_{2}, \cdots, \mathbf{q}_{r}$ are the respective eigenvectors. Here we know the best rank-one approximation of $\mathbf{X}_{k}^{*}$ can be given by $\lambda_{1}^{\prime} \mathbf{q}_{1} \mathbf{q}_{1}^{H}$. Therefore, the candidate solution can be defined as $\tilde{\mathbf{x}}_{k}=\sqrt{\lambda_{1}} \mathbf{q}_{1}$. The other method is to use randomization to extract an approximate QCQP solution from an SDR solution $\mathbf{X}_{k}^{*}$. Considering the $K \times 1$ complex random vector $\boldsymbol{\eta}$ according to the Gaussian distribution with zero mean and covariance $\mathbf{X}_{k}^{*}, \boldsymbol{\eta} \sim \mathcal{C N}\left(\mathbf{0}, \mathbf{X}_{k}^{*}\right)$. Then, with generated random vectors $\boldsymbol{\eta}_{m}, m=1,2, \cdots, M_{\text {random }}$, we normalize $\boldsymbol{\eta}_{m}$ as $\tilde{\mathbf{x}}_{m}=\boldsymbol{\eta}_{m} / \operatorname{norm}\left(\boldsymbol{\eta}_{m}\right)$. Finally, the best solution can be determined as

$$
m^{*}=\arg \min _{m} \max _{l=1,2, \cdots, L}\left|\tilde{\mathbf{x}}_{m}^{T} \mathbf{v}_{l} \mathbf{v}_{l}^{H} \tilde{\mathbf{x}}_{m}^{\dagger}-\chi\right| .
$$

Correspondingly, we obtain the best approximated solution $\hat{\mathbf{x}}_{k}^{*}=\tilde{\mathbf{x}}_{m^{*}}$.

Here we obtain the temporary reconstructed vector with optimal weight $\hat{\mathbf{x}}_{k}^{*}$ by using SDR optimization as

$$
\tilde{\mathbf{s}}_{k}^{*}=\hat{\mathbf{x}}_{k}^{* T} \tilde{\boldsymbol{\Lambda}}^{\frac{1}{2}} \tilde{\mathbf{U}}^{H},
$$

It should be noted that according to (7) and (8), the $\widetilde{\mathbf{s}}_{k}^{*}$ in (15) is not the final estimation of one users' transmitted symbols. Here, we still have two problems to solve

- The estimation $\tilde{\mathbf{s}}_{k}^{*}$ is a phase-rotated version because of taking away the parameter $\theta_{k}$ in (8). Therefore, the first problem is how to determine the parameter $\hat{\theta}_{k}$.

- Although we find out $\tilde{\mathbf{s}}_{k}^{*}$ and then give the phase-correction $\hat{\theta}_{k}$, we still need to know which user the estimation $\tilde{\mathbf{s}}_{k}^{*}$ solved by SDR optimization belongs to.

\subsection{Recognition with embedded training sequences}


It is natural to use a few structured sequences to address the phase-rotation and assignment ambiguity problems which are mentioned above. Since there are $K$ users in our communication scenario, it needs $K$ training sequences $\mathbf{c}_{1}, \mathbf{c}_{2}, \cdots, \mathbf{c}_{K}$ for users with each sequence having length $L^{\prime}$. To successfully determine the index of user, the orthogonality between every two sequences must be assured. A small piece of training sequences is embedded into the transmission frame structure for every user, which results in a slight reduction of the transmission rate by $L^{\prime} / L$. Therefore, the decrease in throughput is negligible. In addition, it should be noted that it is not necessary to have the same start location of all the users' training sequences. In other words, the training sequences in transmission frames could be asynchronous. Based on the estimation $\tilde{\mathbf{s}}_{k}^{*}$ in (15), these training sequences can help us to achieve the following three goals.

1) With the cross correlation processing between the estimation $\widetilde{\mathbf{s}}_{k}^{*}$ and the possible local training sequences $\mathbf{c}_{1}, \mathbf{c}_{2}, \cdots, \mathbf{c}_{K}$, we can determine the user index $k^{\star}$ which corresponds to the maximum correlation peak. This is due to orthogonal and quasiorthogonal properties between training sequences.

2) Meanwhile, we can get the location of the training sequence in the estimation $\tilde{\mathbf{s}}_{k}^{*}$ with searching the maximum correlation peak. We note the starting point of the training sequence $\mathbf{c}_{k^{\star}}$ in $\widetilde{\mathbf{s}}_{k^{\star}}^{*}$ as $l_{\mathrm{st}}$.

3) Perform the phase estimation $\hat{\theta}_{k}$ based on the above results

$$
\hat{\theta}_{k}=\operatorname{angle}\left(\frac{1}{L^{\prime}} \widetilde{\mathbf{s}}_{\left.k^{*}\right|_{l s t} ^{*}}^{*} \mathbf{c}_{k^{*}}^{H}\right),
$$

where $\tilde{\mathbf{s}}_{k^{*} \mid l_{\mathrm{st}}}^{*}=\left[\tilde{\mathbf{s}}_{k}^{*}\left[l_{\mathrm{st}}\right], \tilde{\mathbf{s}}_{k}^{*}\left[l_{\mathrm{st}}+1\right], \cdots, \tilde{\mathbf{s}}_{k}^{*}\left[l_{\mathrm{st}}+L^{\prime}-1\right]\right]$, and here $\tilde{\mathbf{s}}_{k}^{*}\left[l_{\mathrm{st}}+l\right]$ denotes the $\left(l_{\mathrm{st}}+l\right)$-th element of $\tilde{\mathbf{s}}_{k}^{*}$.

Based on the result $\widetilde{\mathbf{s}}_{k}^{*}$ solved by SDR in Section 3.1 and the above procedures, we finally obtain the estimation of user $k^{\star}$ as

$$
\hat{\mathbf{s}}_{k^{*}}=\tilde{\mathbf{s}}_{k}^{*} e^{-j \hat{\theta}_{k}} .
$$

\subsection{Successive extraction for all the users}


It is easy to see that the optimization (P3) in 3.1 and the procedures in 3.2 just give out the estimation $\hat{\mathbf{s}}_{k^{\star}}$ of the $k^{\star}$-th user. Therefore, the final question is how to extract the estimations of the other users' transmitted symbol vectors by using the similar method described in the previous subsections. Actually, we observe that the information from the other users except for the $k^{\star}$-th user is embedded in a new $L \times L$ statistic decision matrix

$$
\boldsymbol{\Sigma}^{\prime}=\boldsymbol{\Sigma}-\hat{\mathbf{s}}_{k^{\star}}^{\dagger} \hat{\mathbf{s}}_{k^{\star}}^{T}
$$

Here, we can consider this problem of extracting the remaining $K-1$ unknown vectors $\left[\hat{\mathbf{s}}_{1}, \hat{\mathbf{s}}_{2}, \cdots, \hat{\mathbf{s}}_{k^{\star}-1}, \hat{\mathbf{s}}_{k^{\star}+1}, \cdots, \hat{\mathbf{s}}_{K}\right]$ as a dimension-reduced problem similar to the previous (P1). Therefore, according to the methods and analysis in 3.1 and 3.2, we can iteratively use the SDR optimization and recognition with remaining embedded training sequences to successively obtain the all users' estimation. Overall, the entire successive SDR-based signal reconstruction can be summarized as the Algorithm 1. The logical relationships between the signal processing modules of the proposed algorithm are also illustrated in Figure 2.

\section{Algorithm 1 Successive Signal Reconstruction with SDR}

Step 1 Given the received signals $\mathbf{Y}$, initialize set $\widehat{\mathbf{S}}=\varnothing$;

Step 2 Build the decision statistic matrix $\mathbf{\Sigma}=\mathbf{Y}^{H} \mathbf{Y} / N$;

Step 3 Eigenvalue decomposition $\boldsymbol{\Sigma}=\mathbf{U} \boldsymbol{\Lambda} \mathbf{U}^{H}$ where $\boldsymbol{\Lambda}=\operatorname{diag} \lambda_{1}, \lambda_{2}, \cdots, \lambda_{L}$ with $\quad \lambda_{1} \geq \cdots \geq \lambda_{L} \quad, \quad \mathbf{U}=\left[\mathbf{u}_{1}, \mathbf{u}_{2}, \cdots, \mathbf{u}_{L}\right] \quad$ with $\quad \boldsymbol{\Sigma} \mathbf{u}_{l}=\lambda_{l} \mathbf{u}_{l} \quad$, $l=1,2, \cdots, L$

Step 4 Selectively construct $\tilde{\boldsymbol{\Lambda}}=\operatorname{diag}\left\{\lambda_{1}, \lambda_{2}, \cdots \lambda_{K}\right\}$ and $\tilde{\mathbf{U}}=\left[\mathbf{u}_{1}, \mathbf{u}_{2}, \cdots, \mathbf{u}_{K}\right]$; Let $\mathbf{V}=\tilde{\boldsymbol{\Lambda}}^{\frac{1}{2}} \tilde{\mathbf{U}}^{H}=\left[\mathbf{v}_{1}, \mathbf{v}_{2}, \cdots, \mathbf{v}_{L}\right], \boldsymbol{\Sigma}^{\prime}=\boldsymbol{\Sigma}$; for $k=1: K$
(a) Solve SDR problem (P3) and obtain $\mathbf{X}_{k}^{*}$;
(b) Extract approximate solution $\hat{\mathbf{x}}_{k}^{*}$ from $\mathbf{X}_{k}^{*}$;
(c) Construct $\widetilde{\mathbf{s}}_{k}^{*}=\hat{\mathbf{x}}_{k}^{* T} \tilde{\mathbf{\Lambda}}^{\frac{1}{2}} \tilde{\mathbf{U}}^{H}$; 
(d) Recognize and determine the user index $k^{\star}$;

(e) Estimate the rotated phase $\hat{\theta}_{k}$;

(f) Reconstruct final estimation $\hat{\mathbf{s}}_{k^{\star}}=\widetilde{\mathbf{s}}_{k}^{*} e^{-j \hat{\theta}_{k}}, \hat{\mathbf{S}}=\hat{\mathbf{S}} \cup \hat{\mathbf{s}}_{k^{\star}}$;

(g) $\boldsymbol{\Sigma}^{\prime}=\boldsymbol{\Sigma}^{\prime}-\hat{\mathbf{s}}_{k^{\star}}^{\dagger} \hat{\mathbf{s}}_{k^{\star}}^{T} ;$

end for

Step 5 Output the set $\hat{\mathbf{S}}$.

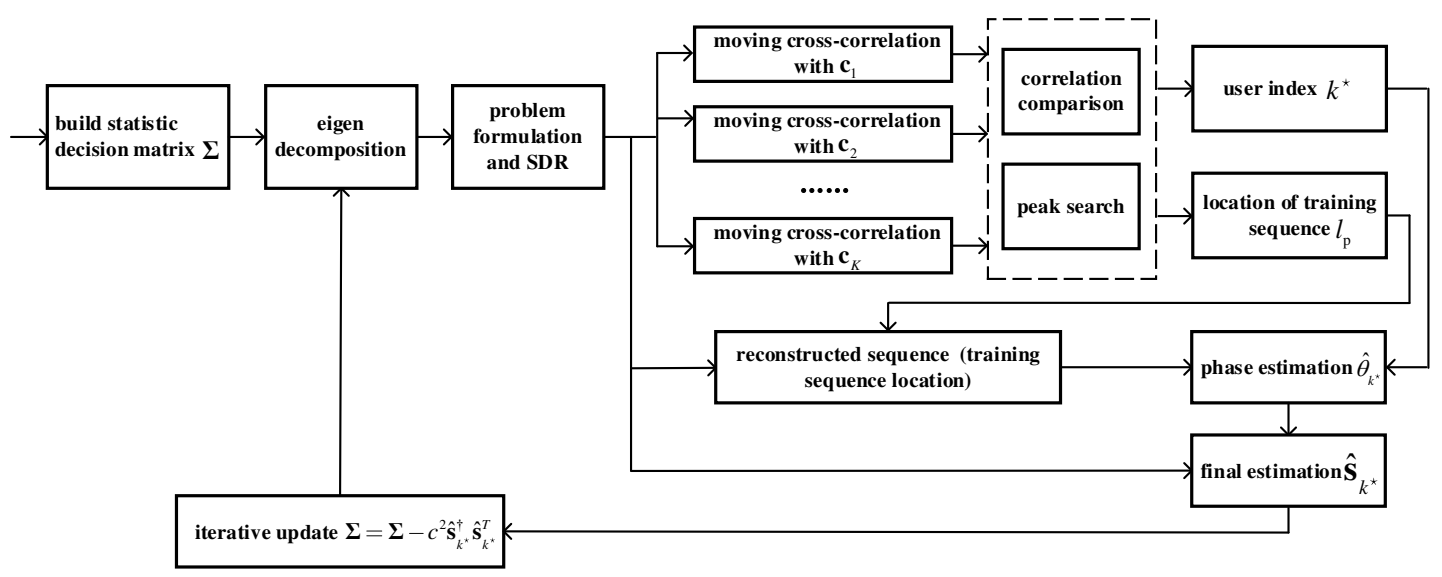

Figure 2: signal processing blocks of the proposed successive signal reconstruction algorithm

\subsection{Complexity Analysis}

The algorithm complexity of the proposed signal reconstruction with SDR is measured in terms of the number of required multiplications. Since the receiver incudes Eigendecomposition and SDR operations, we cannot have an accurate closed expressions to show the overall complexity. We give the complexity analyses of the key operations used in this algorithm in the order of multiplications. At first, we build the signal reconstruction as the problem (P1) expressed in (9), which cannot be solved directly with some algorithm in a polynomial-time complexity. After we convert this problem to (P2) which is a nonconvex QCQP and go further convert it into a SDR problem (P3), feasible solution can be solved in a polynomial time. Table 1 shows the complexity of the key operations. The complexity of construct $\boldsymbol{\Sigma}$ and decomposition $\mathbf{U} \boldsymbol{\Lambda} \mathbf{U}^{H}$ is completely caused by the matrix computation. The complexity of the SDR has been verified as a powerful, computationally efficient approximation technique for many 
nonconvex QCQPs [26], where in the Table 1, $\epsilon$ means a given solution accuracy. The SDR complexity scales slowly (logarithmically) with $\epsilon$ and the general problems do not need very high precision. As for determining $k^{\star}$ and $\hat{\theta}_{k}$, the complexity is mainly caused by the sequence correlation operations, which is related with the length of training sequences $L^{\prime}$. However $L^{\prime}$ is not large because it is chosen according to the number of users, which generally is also much smaller than the number of antennas at receiver.

Table 1: Complexity of the key processing

\begin{tabular}{ll}
\hline Receiver operation & Number of multiplications (Order) \\
\hline decomposition $\mathbf{U} \mathbf{\Lambda} \mathbf{U}^{H}$ & $\mathcal{O} L^{2}$ \\
solve SDR problem (P3) & $\mathcal{O} L^{2} K^{1 / 2} \log (1 / \epsilon)$ \\
determine $k^{\star}$ & $\mathcal{O} K L^{\prime} L-L^{\prime}$ \\
estimate $\hat{\theta}_{k}$ & $\mathcal{O} L^{\prime}$ \\
\hline
\end{tabular}

Table 1 shows the computational complexity comparisons between our proposed scheme and the several existed schemes. For the uplink signal detection schemes with using CSI, the linear detectors, such as zero-forcing (ZF) and minimum mean squared error (MMSE), involves inversion of high dimension matrices which has unacceptable computational complexity. The low-complexity algorithms based on approximate matrix inverse for near-optimal detection in massive MIMO systems are of great interest, e.g., [27-29]. For the uplink signal detection schemes without using CSI, several noncoherent detector have also been extensively researched, such as the energy based scheme [19], DPSK-based scheme [22] and UFC-based noncoherent scheme [24]. Although a piece of training sequences are inserted into the transited symbols in our proposed scheme, they are not used to estimate the CSI at receiver. Therefore, we treat the scheme in this paper as the detection without CSI. From the comparisons in Table 2 , we find the complexity of schemes with CSI is related with the number of users. This is mainly derived from matrix inverse operations, for example the MMSE detector 
$\hat{\mathbf{s}}=\left(\hat{\mathbf{H}}^{H} \hat{\mathbf{H}}+\sigma^{2} \mathbf{I}_{K}\right)^{-1} \hat{\mathbf{H}}^{H} \mathbf{y}$. Comparably, the complexity of schemes without CSI varies greatly according to the different algorithm. Our proposed scheme is mainly related with the length of the received signals. According to the asymptotic property of the massive channels (6) and (7), the large $L$ will improve the performance of our proposed scheme. However, the complexity will also relatively increase. Hence, a trade-off should be made in the practical scenario between the channel coherent time, performance requirement and complexity limitations.

Table 2: Complexity comparison

\begin{tabular}{lll}
\hline & Schemes & Computational complexity \\
\hline \multirow{3}{*}{ with CSI } & MMSE (LS) detection & $\mathcal{O}\left(K^{3}\right)$ \\
& Sequential detection [27] & $\mathcal{O}\left(K^{2}\right)$ \\
& Guass-Seidel method [28] & $\mathcal{O}\left(K^{2}\right)$ \\
& Jacobi method [29] & $\mathcal{O}\left(K^{2}\right)$ \\
\hline \multirow{3}{*}{ Without CSI } & Energy-based scheme [19] & $\mathcal{O}\left(|S|^{K}\right)$ \\
& DPSK-based scheme [22] & $\mathcal{O}\left(|S|^{K}\right)$ \\
& UFC-based scheme [24] & $\mathcal{O}\left(K^{2} N+2^{\log _{2}\left(|\mathcal{S}|^{2}-1\right)} K\right)$ \\
& Proposed scheme & $\mathcal{O} L^{2}+L^{2} K^{1 / 2} \log (1 / \epsilon)$ \\
\hline
\end{tabular}

\section{Results and discussions}

In this section, we carry out several numerical simulations to show how the proposed signal reconstruction scheme works and to examine its statistical performance of the bit error ratio (BER) at the receiver (base-station). We mainly investigate the performance comparisons with different antenna number and the channel quality signal-to-noise ratio $(S N R)$. All the user's transmitted symbols are randomly selected from the same given constellation, and the SDR is solved by the cvx toolbox in MATLAB which is developed by Michael Grant, Stephen Boyd, and Yinyu Ye. We choose the Zadoff-chu sequence as the structured sequences inserted into the transmission frames, which are known as CAZAC (constant amplitude zero autocorrelation) sequences and have 
perfect zeros cross-correlation property [30].

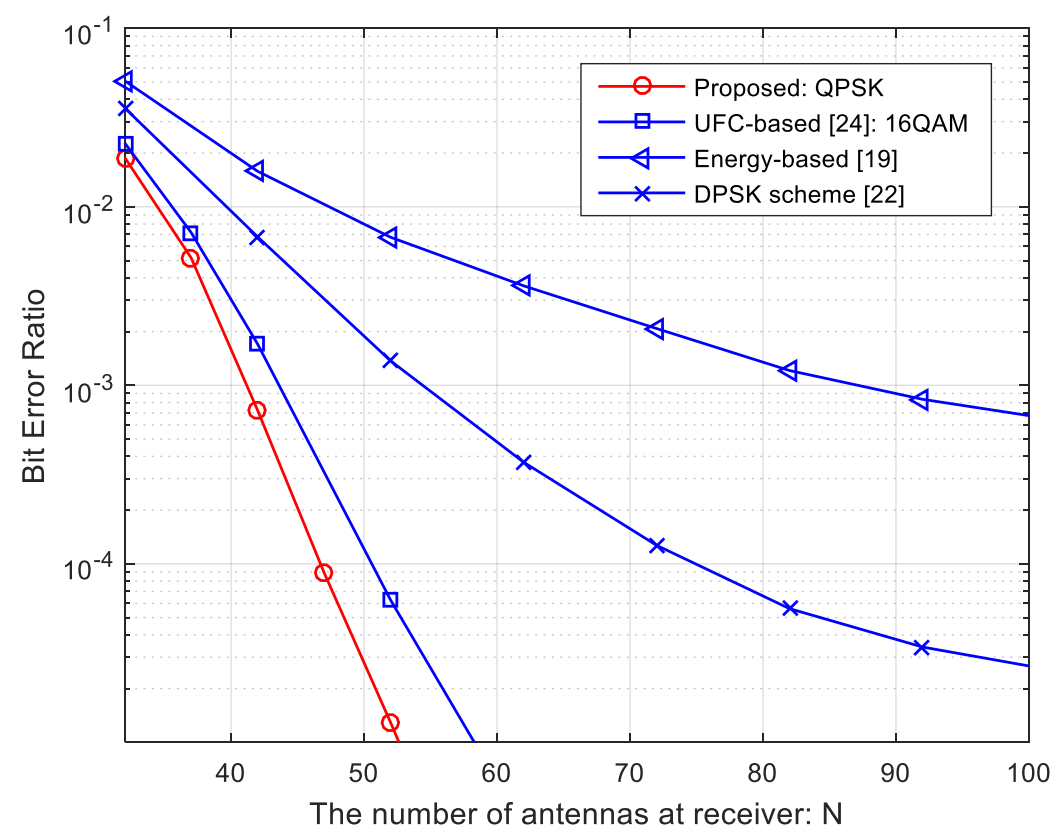

Figure 3: BER performance with increasing the number of antennas at receiver $K=4, L=200$, $\mathrm{SNR}=10 \mathrm{~dB}$.

Figure 3 shows the BER performance with the increasing number of receive antennas. We consider the observation length $L=200$ and channel SNR $=10 \mathrm{~dB}$. Comparisons are also performed with several noncoherent schemes in massive MIMO system, the energy-detection-based noncoherent communication scheme [9], the differential PSK (DPSK) noncoherent scheme [22] and the uniquely factorable constellation (UFC) transmission scheme [24]. From the numerical results, we clearly see that the average BER sharply reduces along with increasing $N$. As for consensus of the simulation condition, we assume the same transmission efficiency, i.e. the number of bits per every channel-use for each user. Obviously, the proposed scheme obtains the minimum BER under the same $\$ \mathrm{~N} \$$ and the same constellation. In addition, we observe BER error floor. This phenomenon is reasonable. Actually, there still exist the channel noises with given $\mathrm{SNR}=10 \mathrm{~dB}$ even when $N$ is very large. 


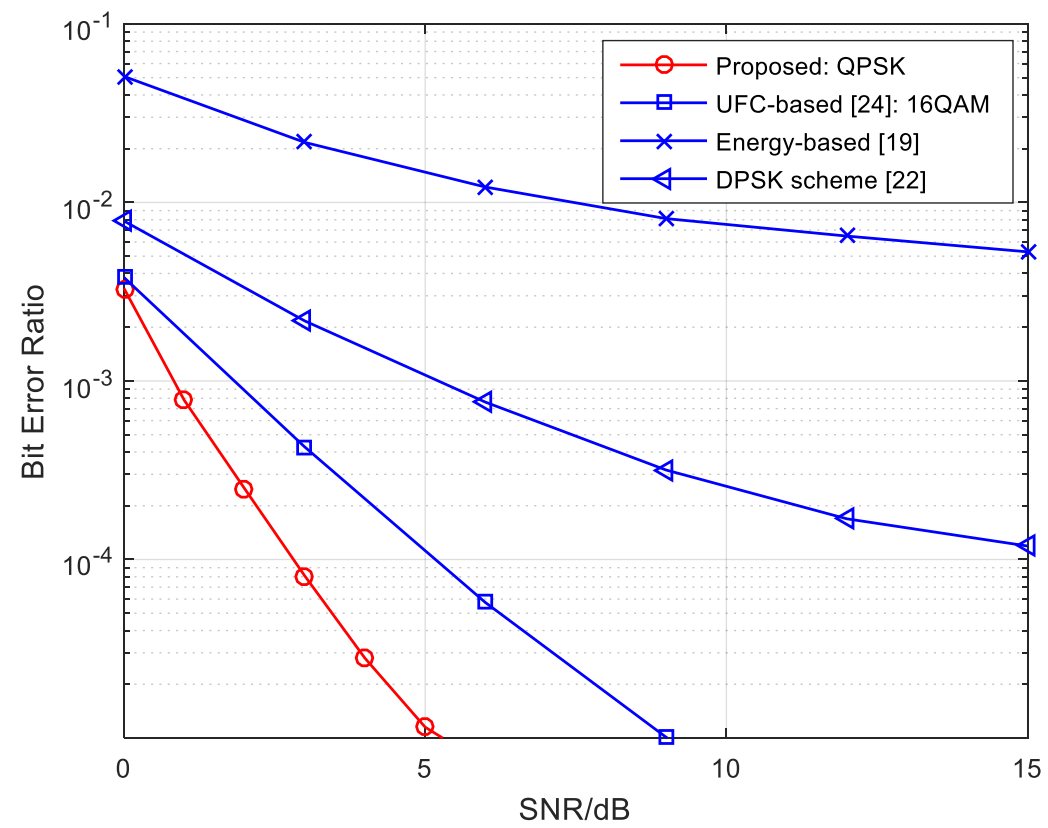

Figure 4: BER performance with increasing $S N R, K=4, L=200, N=64$.

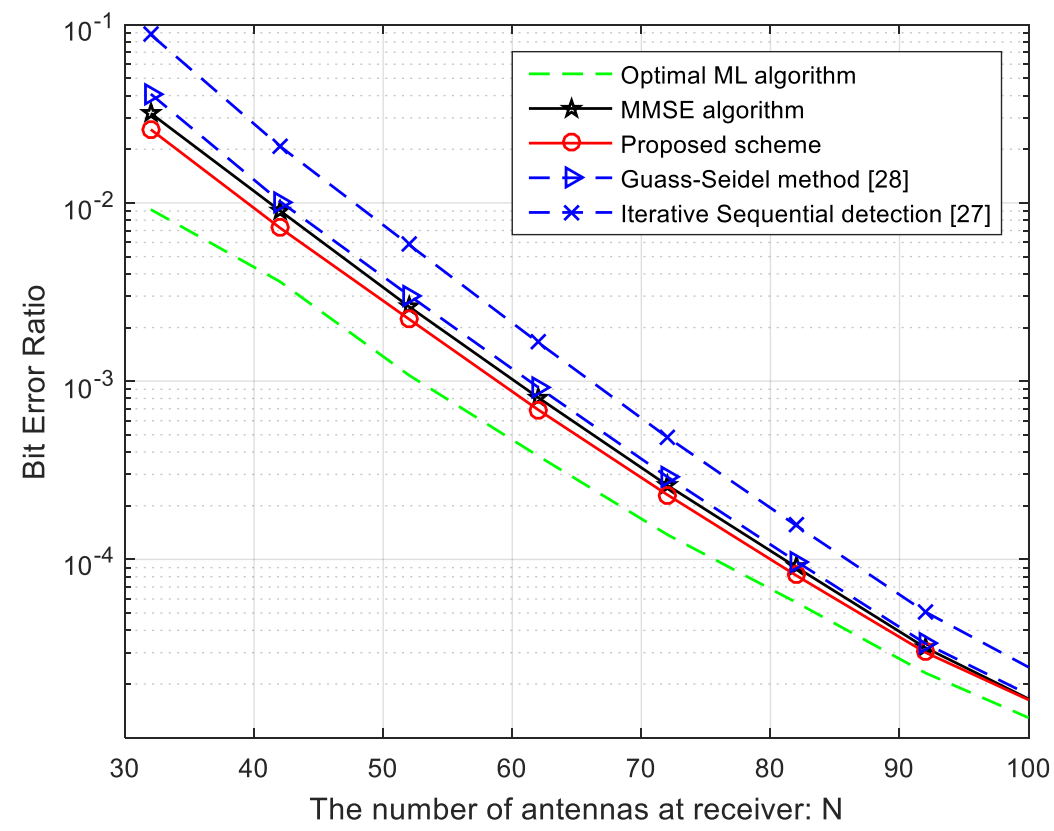

Figure 5: BER performance comparison with ML detector, MMSE detector and typical linear detectors, $\mathrm{K}=4, \mathrm{~L}=200$, SNR $=10 \mathrm{~dB}, 16 \mathrm{QAM}$ constellation.

Figure 4 shows the statistical BER performance with the increasing SNR when the number of antennas is assumed $N=64$. The other simulation parameters are the same as in Figure 3. Compared with noncoherent schemes [19,22,24], the proposed 
signal reconstruction algorithm achieves the competitive performance. In this simulation, we can conclude a high SNR results in much purer decision statistic matrix $\Sigma$ in (4). From the numerical results in Figure 3 and this Figure 4, we can say that $N$ and SNR constitute the two dominant parameter in our proposed algorithm. By comparisons, these noncoherent schemes actually have a strong dependence on a large number of antennas and high SNR to obtain a considerable performance.

Figure 5 gives a performance comparisons between the proposed signal reconstruction algorithm and the detectors with using CSI, i.e., the optimal maximum likelihood (ML), minimum mean squared error (MMSE) detection, the complexityreduced iterative sequential detection [27] and Gauss-Seidel soft-output signal detection [28]. There exist performance gap between our proposed scheme and the optimal ML detection. However, we can see the performance gap gradually decreases with the increasing $N$. In addition, we observe that the proposed algorithm is slightly better than the MMSE detection.

\section{Conclusion}

In summary, we present a signal reconstruction algorithm for the wireless communication scenario with receiver having large number of antennas. The mathematical essence of this algorithm comes from the quasi-orthogonality property of any two independently random vectors when the length of vectors is large according to the probability statistical theory. Therefore, by building a statistical decision matrix with the observed received signals, this property could effectively alleviate the accurate CSI acquirement in the massive MIMO systems. Then, we formulate the original problem of reconstructing the every user's transmitted symbols from the statistical decision matrix and we convert this problem to an SDR optimization problem. With the computationally efficient SDR solver based on the convex optimization theory, we can obtain the estimations successfully. Transmission without the CSI in massive MIMO system is not a new topic. However, most works focus on the transmitting signal design at the transmitter. Our scheme puts the attention on the signal reconstruction based on 
the statistical results of the received signals. By numerical simulations, our proposed scheme shows a relatively competitive performance compared with the coherent linear detection algorithms and the noncoherent transmission strategies.

\begin{abstract}
Abbreviations
MIMO: multiple-input multiple-output; CSI: channel state information; FDD: frequency-division duplex; TDD: time-division duplex; LTE: long term evolution; MRC: maximal ratio combining; IoT: internet of things; SISO: single-input-singleoutput; RF: radio frequency; JSDM: joint spatial division and multiplexing; MUMIMO: multi-user MIMO; SNR: signal-to-noise ratio; SIMO: single-input multipleoutput; MAC: multiple access channels; ASK: amplitude shift keying; PSK: phase shift keying; UFC: uniquely factorable constellation; SDR: semidefinite relaxation; DoF: degree of freedom; QCQPs: Quadratically Constrained Quadratic Programs; ZF: zeroforcing; MMSE: minimum mean squared error; BER: bit error ratio; SNR: signal-tonoise ratio; CAZAC: constant amplitude zero autocorrelation; DPSK: differential phase shift keying; ML: maximum likelihood.
\end{abstract}

\title{
Availability of data and materials
}

We decided that the data does not need to be shared since all data have been obtained through the simulation using the MATLAB program.

\section{Competing interests}

The authors declare that they have no competing interests.

\section{Funding}

This work was supported in part by China's Postdoctoral Grants No. 2018M640958, Youth Program of National Natural Science Foundation of China Grants No. 61901325, Natural Science Foundation of Shaanxi Province under Grants No. 2019JQ-658 and Fundamental Research Funds for the Central Universities Grants No. 8002/20101196698.

\section{Author's contributions}

Author Guo Li was in full charge of the major theoretical analysis, algorithm design, experimental simulations and the manuscript writing. 


\section{Acknowledgements}

Not applicable

\section{References}

[1] T. L. Marzetta, "Noncooperative cellular wireless with unlimited numbers of base station antennas,” IEEE Trans. Wireless Commun., vol. 9, pp. 3590-3600, May 2010.

[2] F. Rusek, D. Persson, and B. K. Larsson, "Scaling up MIMO: opportunities and challenges with very large arrays," IEEE Signal Process. Mag., vol. 30, pp. 40-60, Sep. 2013.

[3] E. G. Larsson, O. Edfors, F. Tufvesson, and T. L. Marzetta, "Massive MIMO for next generation wireless systems," IEEE Commun. Mag., vol. 52, no. 2, pp. 186-195, Feb. 2014.

[4] P. Liu, S. Jin, T. Jiang, Q. Zhang, and M. Matthaiou, "Pilot power allocation through user grouping in multi-cell massive MIMO systems," IEEE Trans. Commun., vol. 65, no. 4, pp. 1561-1574, Apr. 2017.

[5] S. Jin, X. Wang, Z. Li, K.-K. Wong, Y. Huang, and X. Tang, "On massive MIMO zero-forcing transceiver using time-shifted pilots,” IEEE Trans. Veh. Technol., vol. 65, no. 1, pp. 59-74, Jan. 2016.

[6] F. Kaltenberger, H. Jiang, M. Guillaud, and R. Knopp, "Relative channel reciprocity calibration in MIMO/TDD systems," in Proc. Future Network and Mobile Summit, pp. 1-10, IEEE, Mar. 2010.

[7] 3GPP, "6.10.1.2 mapping to resource elements," Available: http://www.3gpp.org/ftp/Specs/html-info/36211.htm, Dec. 2010.

[8] A. F. Molisch, V. V. Ratnam, S. Han, Z. Li, S. L. H. Nguyen, L. Li, and K. Haneda, "Hybrid Beamforming for Massive MIMO A Survey," IEEE Commun. Mag., vol. 55, no. 9, pp. 134141, Sep. 2017

[9] A. Adhikary, J. Nam, J. -Y Ahn, and G. Caire, "Joint spatial division and multiplexing: the large-scale array regime," IEEE Trans. Inf. Theory, vol. 59, no. 10, pp. 6441- 6463, Oct. 2013.

[10] J. Nam, A. Adhikary, J. -Y Ahn, and G. Caire, "Joint spatial division and multiplexing: Opportunistic beamforming, user grouping and simplified downlink scheduling," IEEE J. Sel. Areas Commun., vol. 8, no. 5, pp. 876 - 890, Mar. 2014.

[11] C. Sun, X. Gao, S. Jin, M. Matthaiou, Z. Ding, and C. Xiao, "Beam division multiple access transmission for massive MIMO communications," IEEE Trans. Commun., vol. 63, no. 6, Jun. 2015.

[12] A. Liu and V. Lau, "Phase only RF precoding for massive MIMO systems with limited RF chains,” IEEE Trans. Signal Process., vol. 62, no. 17, pp. 4505-4515, Sep. 2014.

[13] Z. Gao, L. Dai, Z. Wang and S. Chen, "Spatially Common Sparsity Based Adaptive Channel Estimation and Feedback for FDD Massive MIMO,” IEEE Trans. Signal Process., vol. 63, no. 23, pp. 6169-6183, Dec. 2015.

[14] D. G. Yang, W. and E. Riegler, "On the capacity of large-MIMO block-fading channels," IEEE J. Sel. Areas Commun., vol. 31, pp. 117-132, Jun. 2013.

[15] J. Wang, Y. Zhao, and S.F. Fan, "Unitary space-time modulation of turbo trellis-coded for multiple-antenna Rayleigh fading channel," in Proc. Wireless Communications Networking and Mobile Computing (WCNC), pp. 72--76, IEEE, Oct. 2007.

[16] L. Zheng and D. Tse, "Communication on the Grassmann manifold: a geometric approach to the noncoherent multiple-antenna channel," IEEE Trans. Inform. Theory, vol. 48, pp. 359-383, 
Mar. 2002.

[17] B. M. Hochwald and T. L. Marzetta, "Unitary space-time modulation for multiple-antenna communications in Rayleigh flat fading," IEEE Trans. Inform. Theory, vol. 46, pp. 543-564, Mar. 2000.

[18] M. Brehler and M. K. Varanasi, "Asymptotic error probability analysis of quadratic receivers in Rayleigh-fading channels with applications to a unified analysis of coherent and noncoherent space-time receivers," IEEE Trans. Inform. Theory, vol. 47, pp. 2383-2399, Jul. 2001.

[19] A. Manolakos, M. Chowdhury, and A. J. Goldsmith, "Constellation design in noncoherent massive $\{\mathrm{SIMO}\}$ systems," in Proc. IEEE Global Communications Conference (GLOBECOM), pp. 3690-3695, Dec. 2014.

[20] M. Chowdhury, A. Manolakos, and A. Goldsmith, "Coherent and noncoherent schemes for massive $\{$ SIMO $\}$ systems," in Proc. IEEE International Conference on Communications (ICC), May 2015.

[21] M. Chowdhury, A. Manolakos, and A. Goldsmith, "Scaling laws for noncoherent energy-based communications in the SIMO MAC," IEEE Trans. Inform. Theory, vol. 62, no. 4, pp. 19801992, Feb. 2016.

[22] A. G. Armada and L. Hanzo, "A noncoherent multi-user large scale SIMO system relaying on M-ary DPSK," in Proc IEEE International Conference on Communications (ICC), pp. 25172522, Jun. 2015.

[23] G. Li and F. Gong, "Space-time uplink transmission in noncoherent systems with receiver having massive antennas," IEEE Commun. Lett., vol. 21, no. 4, pp. 929-932, 2017.

[24] L. Xiong and J. K. Zhang, "Energy-efficient uniquely factorable constellation designs for noncoherent SIMO channels," IEEE Trans. Veh. Technol., vol. 61, no.5, pp. 2130-2144, Jun. 2012.

[25] H. Cramer, "Random variables and probability distributions," vol. 36, Cambridge University Press, 2004.

[26] Z.-Q. Luo, W.-K. Ma, A. M.-C. So, Y. Ye, and S. Zhang, "Semidefinite relaxation of quadratic optimization problems," IEEE Signal Process. Mag., vol. 20, pp. 1-15, May 2010.

[27] M. Mandloi and V. Bhatia, "Low-complexity near-optimal iterative sequential detection for uplink massive MIMO systems," IEEE Commun. Lett., vol. 21, pp. 568-571, Mar. 2017.

[28] L. Dai, X. Gao, X. Su, S. Han, I. Chih-Lin and Z. Wang, "Low complexity soft-output signal detection based on Gauss-Seidel method for uplink multiuser large-scale MIMO systems," IEEE Trans. Veh. Technol., vol. 64, pp. 4839-4845, Oct. 2015.

[29] X. Qin, Z. Yan and G. He, "A near-optimal detection scheme based on joint steepest descent and Jacobi method for uplink massive MIMO systems," IEEE Commun. Lett., vol. 20, pp. 276 - 279, Dec. 2015.

[30] L. Song, and J. Shen, "Evolved Cellular Network Planning and Optimization for UMTS and LTE,” CRC Press, New York, 2011.

\section{Figures}




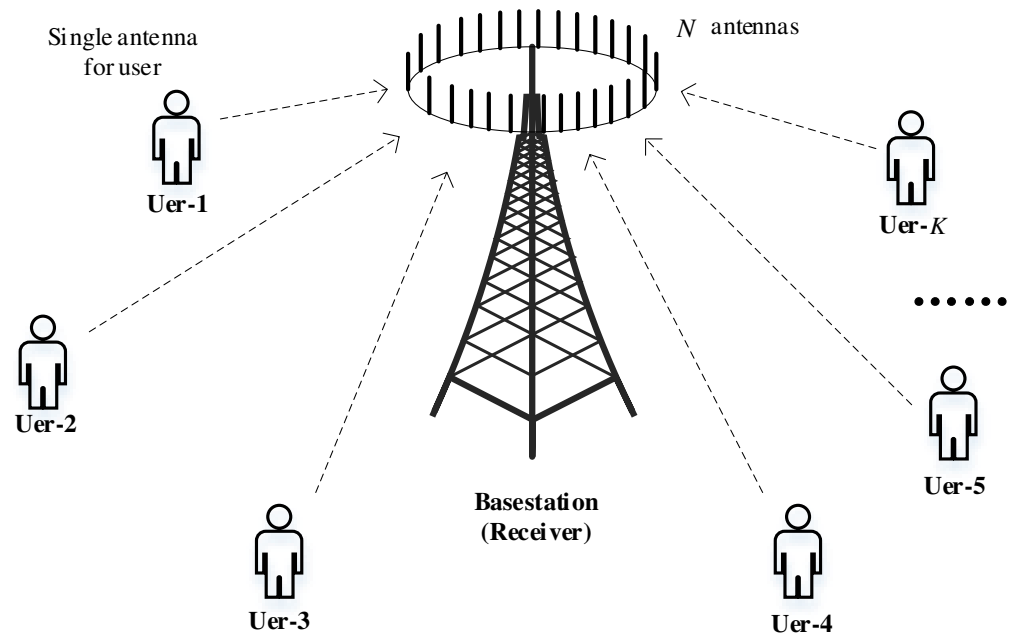

Figure 1: Scenario with multiple users and a based station having large number of antennas.

We consider $K$ single-antenna transmitters (Users) and one receiver (Base-station) deployed with $N$ antennas, $N \gg K$. 
Figure 2: Signal processing blocks of the proposed successive signal reconstruction algorithm.

The logical relationships between the signal processing modules of the proposed algorithm: Successive Signal Reconstruction with SDR. 


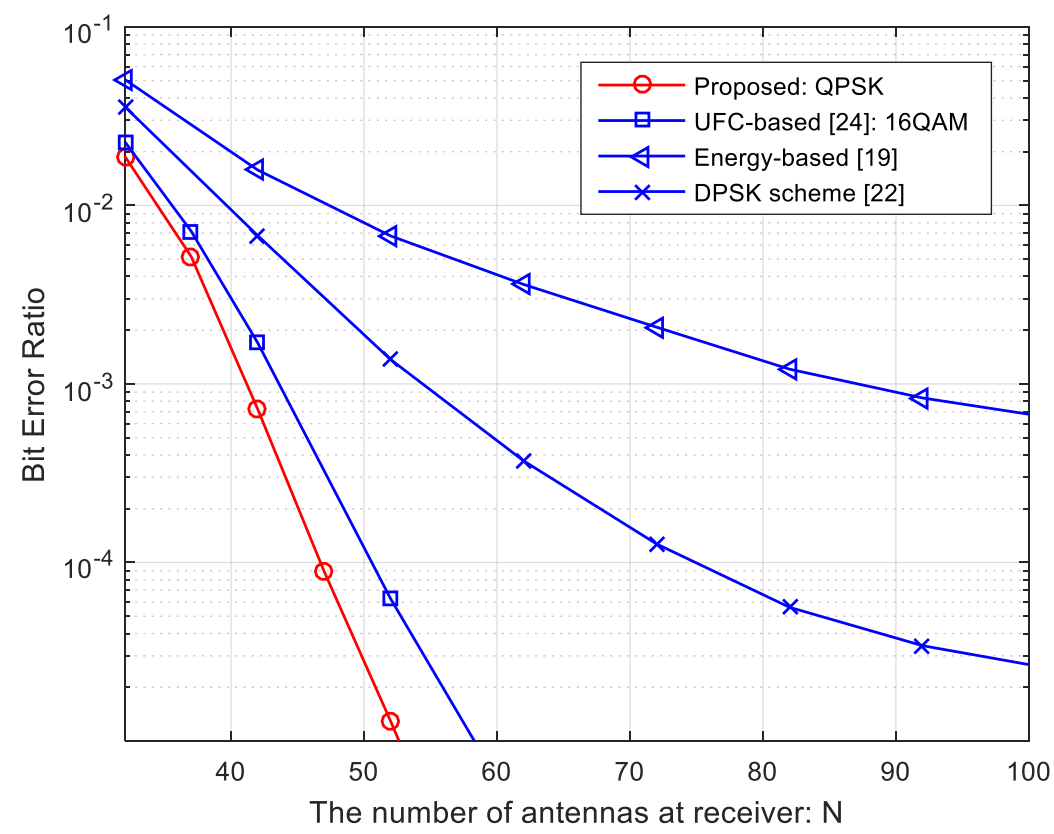

Figure 3: BER performance with increasing the number of antennas at receiver. We consider the observation length $L=200$ and channel SNR $=10 \mathrm{~dB}$. Comparisons are also performed with several noncoherent schemes in massive MIMO system, the energy-detection-based noncoherent communication scheme [19], the differential PSK (DPSK) noncoherent scheme [22] and the uniquely factorable constellation (UFC) transmission scheme [24]. From the numerical results, we clearly see that the average BER sharply reduces along with increasing $N$. As for consensus of the simulation condition, we assume the same transmission efficiency, i.e. the number of bits per every channel-use for each user. Obviously, the proposed scheme obtains the minimum BER under the same \$N\$ and the same constellation. In addition, we observe BER error floor. This phenomenon is reasonable. Actually, there still exist the channel noises with given $\mathrm{SNR}=10 \mathrm{~dB}$ even when $N$ is very large. 


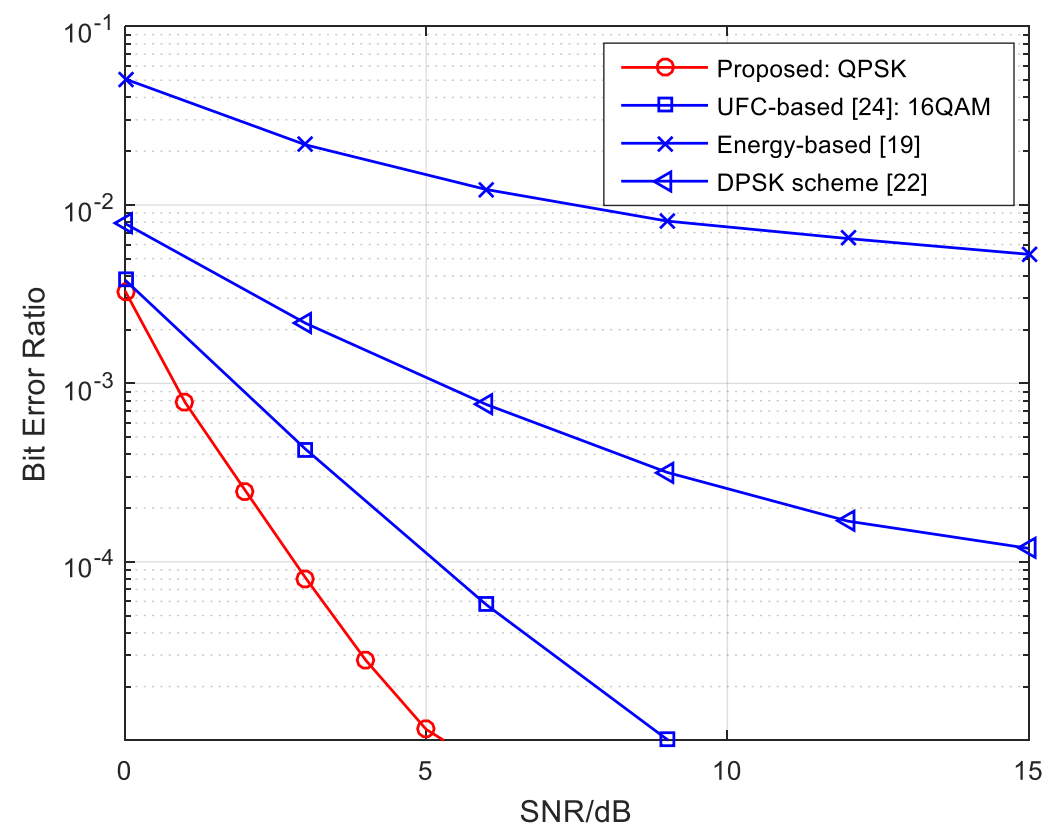

Figure 4: BER performance with increasing SNR. Compared with noncoherent schemes $[19,22,24]$, the proposed signal reconstruction algorithm achieves the competitive performance. In this simulation, we can conclude a high SNR results in much purer decision statistic matrix $\boldsymbol{\Sigma}$ in (4). From the numerical results in Figure 3 and this Figure 4, we can say that $N$ and SNR constitute the two dominant parameter in our proposed algorithm. By comparisons, these noncoherent schemes actually have a strong dependence on a large number of antennas and high SNR to obtain a considerable performance. 


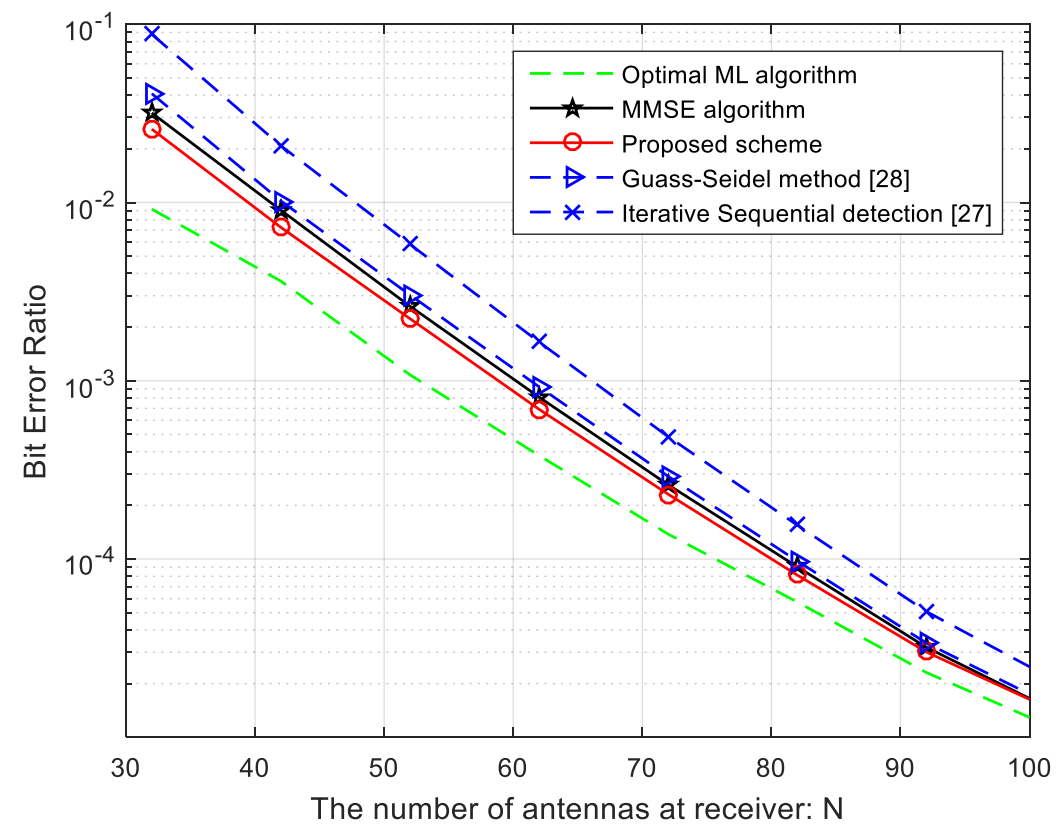

Figure 5: BER performance comparison with ML detector, MMSE detector and typical linear

detectors. The performance comparisons between the proposed signal reconstruction algorithm and the detectors with using CSI, i.e., the optimal maximum likelihood (ML), minimum mean squared error (MMSE) detection, the complexity-reduced iterative sequential detection and Gauss-Seidel soft-output signal detection. There exist performance gap between our proposed scheme and the optimal ML detection. However, we can see the performance gap gradually decreases with the increasing $N$. In addition, we observe that the proposed algorithm is slightly better than the MMSE detection. 


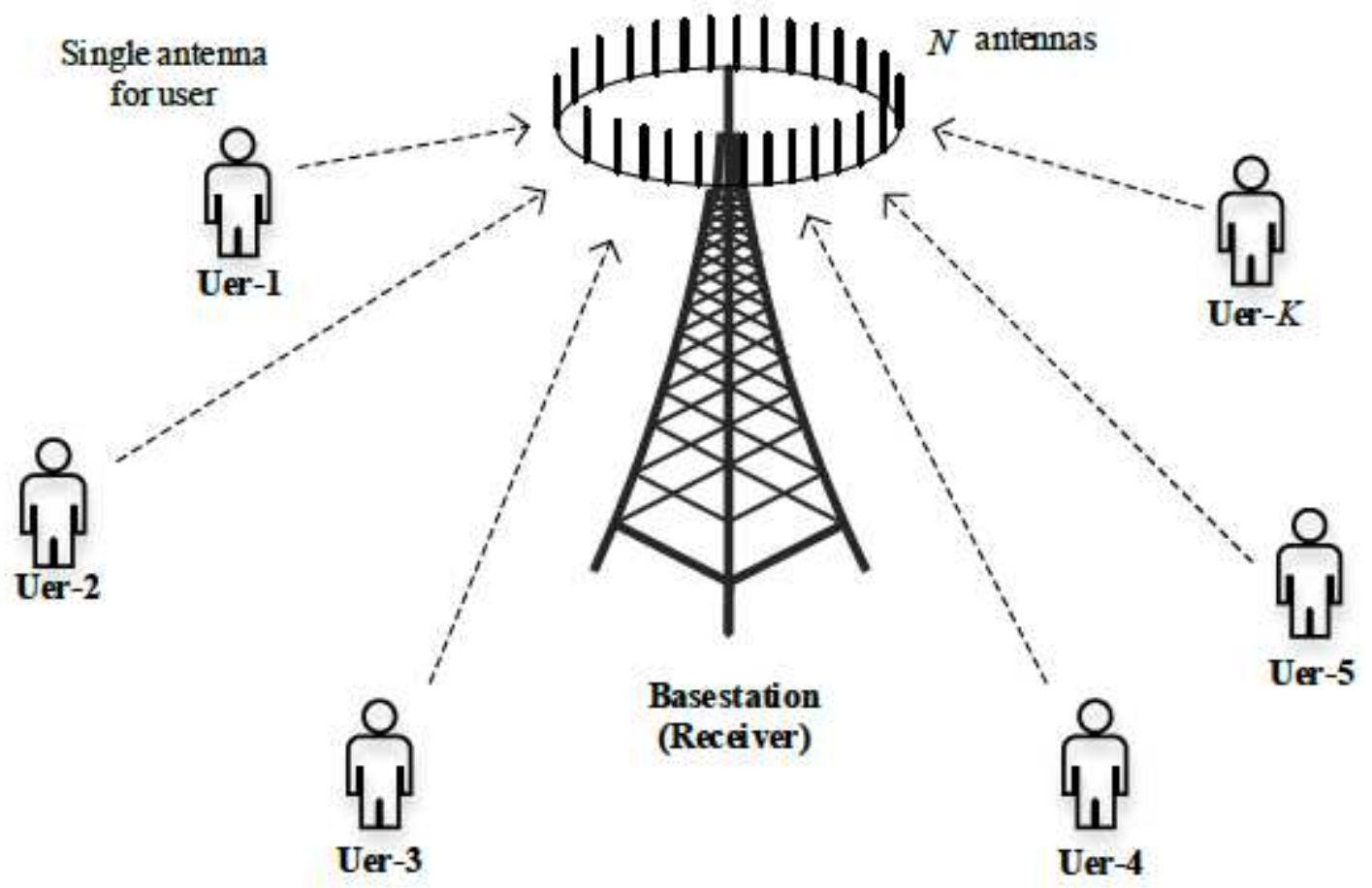

Figure 1

Scenario with multiple users and a based station having large number of antennas

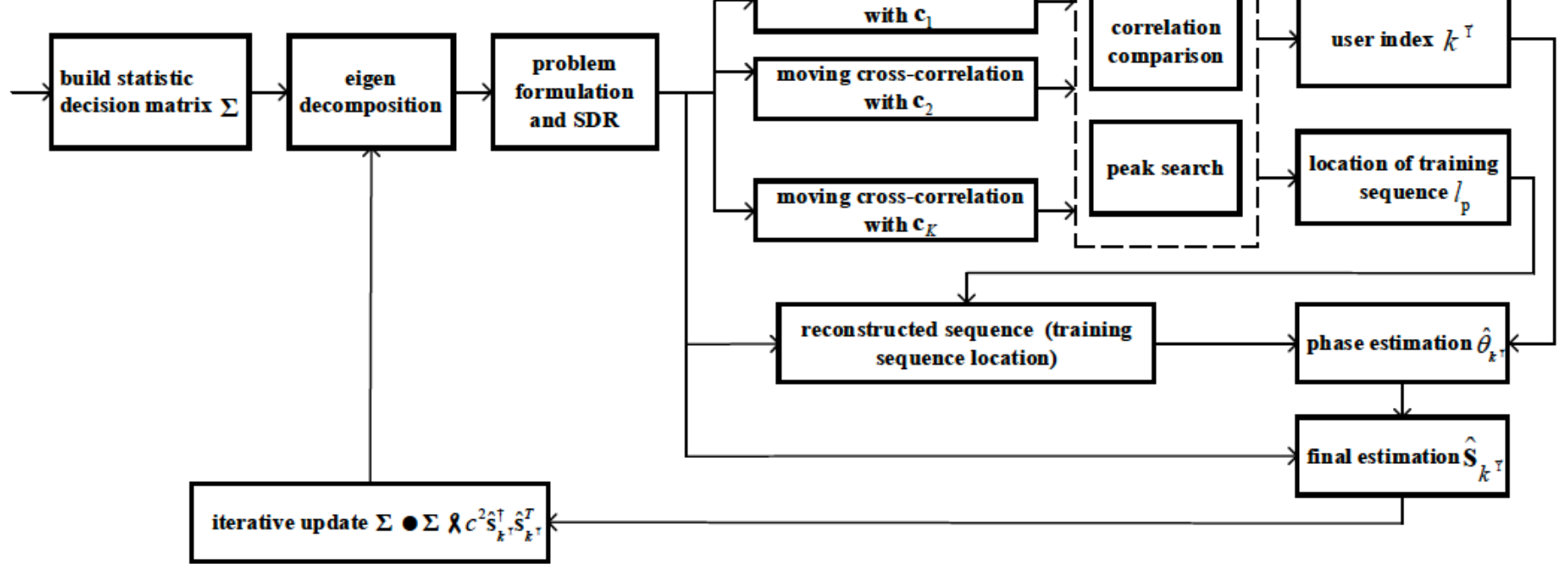

Figure 2

signal processing blocks of the proposed successive signal reconstruction algorithm 


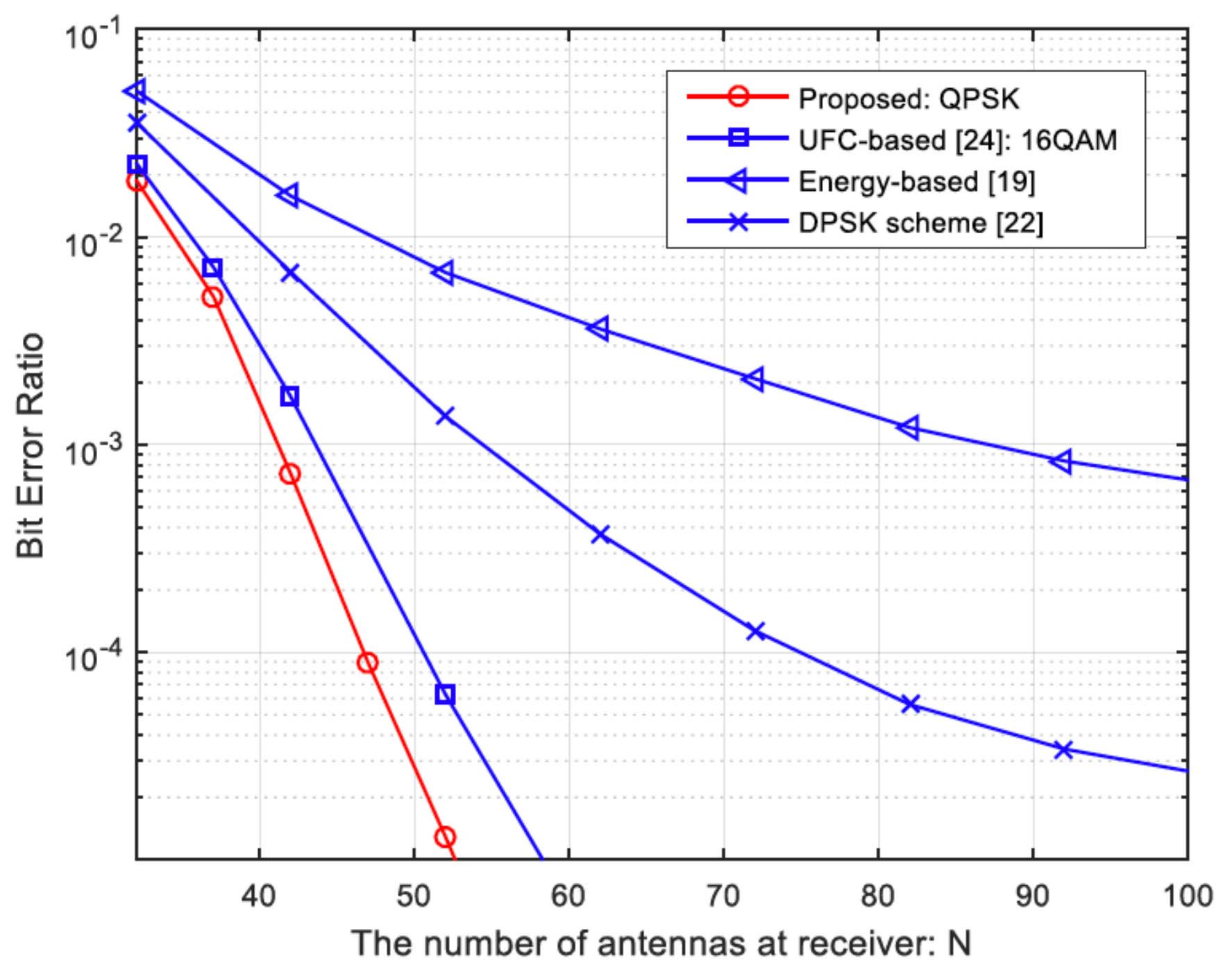

Figure 3

BER performance with increasing the number of antennas at receiver $K=4, L=200, S N R=10 d B$. 


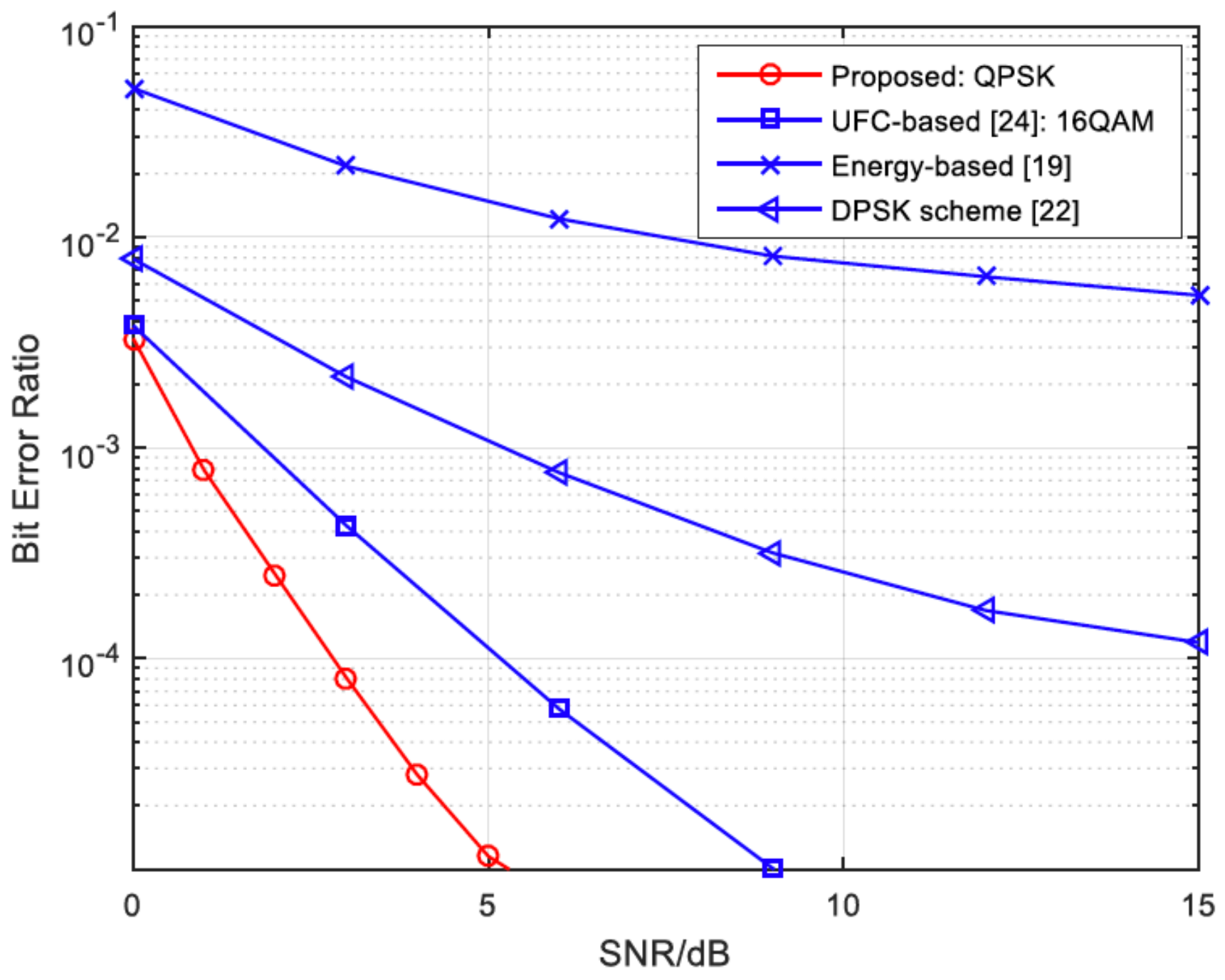

Figure 4

BER performance with increasing $S N R, K=4, L=200, N=64$. 


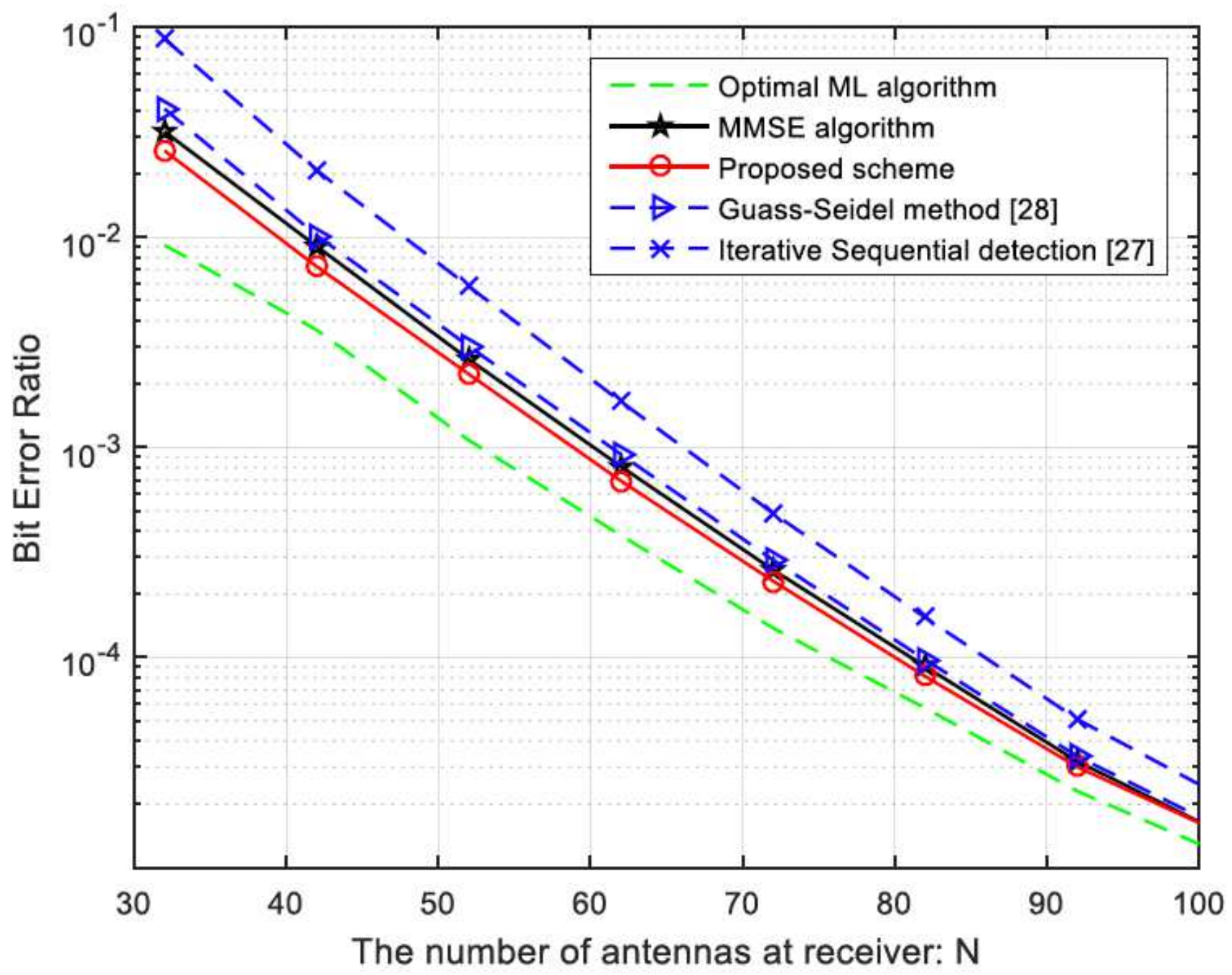

Figure 5

BER performance comparison with ML detector, MMSE detector and typical linear detectors, $K=4$, $L=$ $200, \mathrm{SNR}=10 \mathrm{~dB}, 16 \mathrm{QAM}$ constellation. 